$81 \mid 2012$

Pouvoirs du mythe dans les littératures francophones du Maghreb et du Machrek

\title{
Deux mythes féminins du Maghreb : la Kahina et Aïcha Kandicha
}

Samira Douider

\section{OpenEdition}

\section{Journals}

Édition électronique

URL : http://journals.openedition.org/recherchestravaux/547

DOI : 10.4000/recherchestravaux.547

ISSN : 1969-6434

Éditeur

UGA Éditions/Université Grenoble Alpes

Édition imprimée

Date de publication : 30 décembre 2012

Pagination : 75-81

ISBN : 978-2-84310-238-7

ISSN : 0151-1874

\section{Référence électronique}

Samira Douider, «Deux mythes féminins du Maghreb : la Kahina et Aïcha Kandicha », Recherches \& Travaux [En ligne], 81 | 2012, mis en ligne le 30 juin 2014, consulté le 10 décembre 2020. URL : http:// journals.openedition.org/recherchestravaux/547 ; DOI : https://doi.org/10.4000/recherchestravaux. 547 


\section{Deux mythes féminins du Maghreb : la Kahina et Aïcha Kandicha}

Les mythes, de par leur origine ancienne, contribuent à la construction des sociétés. Ils permettent d'expliquer le monde et de justifier certains comportements ou situations. Nombreux sont ceux qui parcourent le monde maghrébin, donnant naissance à des personnages qui hantent la mémoire collective. Deux figures féminines qui traversent l'histoire culturelle du Maghreb ont marqué leur époque par leur personnalité extraordinaire et leurs actes fabuleux au point d'en devenir des mythes par les symboles qu'elles transmettent : la Kahina et Aïcha Kandicha. L'évocation seule de ces deux noms provoque l'attirance vis-à-vis de l'une et le recul vis-à-vis de l'autre. Ces deux femmes se ressemblent sur bien des points, mais les mémoires collectives ont plutôt retenu, de l'une, le caractère glorieux et, de l'autre, la terreur qu'elle a provoquée.

D'appartenance maghrébine, la Kahina est connue à travers toute l'Afrique du Nord, alors qu'Aïcha Kandicha est principalement associée au Maroc et parfois à l'Ouest algérien. L'histoire n'est pas unanime sur les événements de leurs vies dont on connaît plusieurs variantes. En croisant les diverses versions, il ressort que toutes les deux ont subjugué par leur beauté et particulièrement celle de leur chevelure dont il est dit, en ce qui concerne la Kahina, qu'elle est "éployée comme les ailes de l'aigle ${ }^{\mathrm{I}}$ ", mais également par leur force de caractère qui les mènera jusqu'au bout de leurs convictions et jusqu’à la mort.

I. G. Camps, L'Afrique du Nord au féminin. Hérö̈nes du Maghreb et du Sahara, Perrin, I992, p. I26. 


\section{De l'histoire à la légende}

La première, la Kahina, personnage historique, est une princesse berbère qui s'est opposée, au $\mathrm{VII}^{\mathrm{e}}$ siècle, aux Arabes et à l'implantation de l'islam lors de l'invasion du Maghreb par les troupes d'Hassan. L'histoire retient d'elle qu'elle a rassemblé les différentes tribus contre l'envahisseur; grâce à cette union, les Berbères ont réussi à arrêter les troupes arabes et à les renvoyer en Tripolitaine. Son nom, qui est en fait un surnom et signifie «la prophétesse», lui accorde des pouvoirs surnaturels de devineresse qui vont nourrir sa légende. La seconde, Aïcha Kandicha, que l'on retrouve au Maroc et dans l'Ouest de l'Algérie, est connue sous différentes appellations : Aïcha Quendicha (Kendicha), Lalla Aïcha, Aïcha Soudaniyya, Aïcha l'gnaouia, Aïcha la contessa. Ces différents surnoms soulignent les diverses origines de cette figure $^{2}$. Il s'agirait - personnage semi-légendaire - d'une femme d'une beauté incomparable dont l'origine est multiple (berbère, portugaise et soudanaise), vêtue de belles étoffes dissimulant ses seins pendants et ses pieds de chameau, de chèvre ou de mule. Originaire d'El Jadida, elle aurait, au $\mathrm{XVI}^{\mathrm{e}}$ siècle, contribué à combattre les envahisseurs portugais. Sa technique consistait à utiliser ses charmes pour attirer les soldats qui étaient ensuite tués par ses complices. Les colonisateurs, pour la punir, auraient exécuté toute sa famille ainsi que son fiancé. Choquée, la jeune femme serait devenue folle et aurait erré dans la forêt. Le bruit courut auprès des populations locales qu'elle s'attaquait aux jeunes gens pour les dévorer.

Ces différentes versions ont laissé des traces dans les mémoires collectives et ont stéréotypé l'image de ces femmes. La Kahina est considérée comme la femme qui a défendu son territoire contre l'envahisseur. Guerrière exceptionnelle, forte, qui protège les siens, elle laisse le souvenir d'une bienfaitrice. Aïcha Kandicha, quant à elle, demeure avec l'image trouble d'un être variant de l'humain à l'animal et tentant de séduire pour mieux détruire. On l'évoque, de nos jours encore, pour faire peur aux enfants. Souvent décrite comme attaquant les voyageurs égarés ou détournant les hommes de leurs épouses, elle correspond à la fois à la femme fatale qui encourage les fantasmes masculins mais également à la mère phallique, ce qui relance les fantasmes féminins. Le côté sexuel contribue à accentuer le caractère obscur de cette femme. Cependant, bien qu'elle soit très crainte, elle apparait parfois comme protectrice.

Pourquoi le mythe a-t-il été positif pour la Kahina et négatif pour Aïcha Kandicha? Comment ces mythes sont-ils encore utilisés dans les différentes

2. Le mot kandicha viendrait de l'espagnol contessa. 
expressions artistiques actuelles? Pour essayer de comprendre cette différence de traitement, nous pouvons nous reporter au dernier épisode de leurs vies supposées. La Kahina, encouragant la politique de la terre brûlée, aurait retourné les autochtones contre elle au point de se faire massacrer. L'endroit où elle serait morte retient la mémoire de son nom "Bir El Kahina». Elle a donc subi la vindicte populaire de manière injuste puisque, malgré ses actes, elle a sauvé le pays. Cette injustice, exercée à son encontre, a certainement conforté l'image de grandeur de la Kahina qui n'a pas été violente envers les populations autochtones. À l'inverse, Aïcha Kandicha, à la perte de son fiancé et peut-être de sa famille (selon la version), s'est retournée contre les siens et a commencé à s'attaquer à eux. Apparemment déconsidérée par ses actes, elle serait alors apparue comme un danger aux yeux de ses partisans. Il semble que la Kahina a persisté dans les mémoires collectives comme le symbole de la résistance contre l'ennemi, alors qu'Aïcha Kandicha représente, pour tous, la femme tentatrice qui attire les hommes et les dévore. Ainsi, seul un pan de l'histoire est resté dans les mémoires, ce qui a créé une image différente de ces deux femmes qui, pourtant, toutes les deux se sont opposées aux envahisseurs. Manifestement, c'est la sexualité qui joue un rôle essentiel pour opposer les deux mythes.

\section{Littérature, cinéma, musique}

C'est principalement la littérature qui utilise ces figures; de nombreux essayistes, désirant traiter de la question de la femme algérienne ou maghrébine, ont utilisé le personnage de la Kahina afin de symboliser la résistance féminine. C'est le cas de Gisèle Halimi dans son livre La Kahina ${ }^{3}$ ou de Baya Jurquet-Bouhoune dans Femmes algériennes : de la Kahina au code de la famille ${ }^{4}$ ou encore de Denise Brahimi dans Femmes arabes et sours musulmanes'. Ces écrivains militent pour la libération des femmes et, dans leurs écrits, le personnage de la Kahina est un moyen de transmettre leur message. Gisèle Halimi réécrit l'histoire de la Kahina dans un roman parfois controversé en raison de ses interprétations de l'Histoire. Baya Jurquet-Bouhoune s'élève, dans son essai, contre les lois qui vont à l'encontre des femmes algériennes et particulièrement dans le code de la famille. Elle rend hommage au courage de celles qui ont combattu, le plus souvent anonymement, pour leur libération.

3. G. Halimi, La Kahina, Plon, 2006.

4. B. Jurquet-Bouhoune, J. Jurquet, Femmes algériennes : de la Kahina au code de la famille, Le Temps des cerises, 2007.

5. D. Brahimi, Femmes arabes et sours musulmanes, Tierce, 1984. 
Denise Brahimi, dans Femmes arabes et soeurs musulmanes, trace un panorama de figures féminines qui ont marqué le monde arabo-musulman. La Kahina trouve donc légitimement sa place dans ces écrits du fait de son impact dans l'histoire des femmes.

En littérature, la Kahina est présente autant chez des auteurs marocains, algériens que tunisiens. Cette héroïne apparaît, en effet, comme la Maghrébine par excellence, puisque, à l'époque où elle vécut, les frontières des différents pays du Maghreb n'étaient pas délimitées et que son histoire l'a menée de la Tunisie au Maroc en passant par l'Algérie : chacun des peuples des trois pays la revendiquant comme étant sienne. De plus, symbole de la résistance berbère face au monde arabo-musulman, elle est donc le plus souvent utilisée par des auteurs qui défendent la cause berbère tels que l'Algérien Kateb Yacine ou le Marocain Mohammed Khaïr-Eddine. Mohammed-Saâd Zemmouri souligne dans son étude portant sur Présence berbère et nostalgie païenne dans la littérature maghrébine de langue française que "Ce personnage-symbole semble hanter l'imaginaire des auteurs qui écrivent pour la cause berbère ${ }^{6} »$ et d'autre part que :

$\mathrm{Si}$ tous ces écrivains revendiquent sans détour cette figure historico-légendaire, chacun en construit dans ses textes une image personnelle dans laquelle on perçoit le travail de l'imaginaire du poète qui élabore autour de l'héroïne un mythe'.

Kateb Yacine, est particulièrement attaché à cette figure féminine historique qui apparaît dans certains de ses écrits. Dans son premier roman, Nedjma, il n'introduit pas directement le personnage de la Kahina, mais il le fait symboliquement, retenant d'elle l'élément qui la caractérise par excellence : la résistance. Dans ce roman, l'hérö̈ne incarne l'Algérie qui ne veut pas se laisser prendre par l'envahisseur et qui donc se bat. Certaines similitudes sont à noter entre Nedjma et la Kahina : amours tumultueuses et objets de désir :

Nedjma qu'aucun époux ne pouvait apprivoiser, Nedjma l'ogresse au sang obscur [...], l'ogresse qui mourut de faim après avoir mangé ses trois frères [...], Nedjma la goutte d'eau trouble qui entraîna Rachid hors de son rocher ${ }^{8}$.

Kateb Yacine accentue dans ce passage le caractère sexuel de la Kahina. Par l'assimilation à une ogresse, cette description rappelle le personnage d'Aïcha Kandicha dont la sexualité est considérée comme hors norme.

6. M.-S. Zemmouri, Présence berbère et nostalgie païenne dans la littérature maghrébine de langue française, Publications de la Faculté des lettres et des sciences humaines de Tétouan, 2000, p. 97.

7. Ibid., p. 97.

8. Kateb Yacine, Nedjma, Seuil, I956, p. I80. 
La Kahina apparaît à nouveau, en 1974, dans une pièce intitulée $L a$ Guerre de deux mille ans, dans laquelle le personnage est clairement évoqué, sous son véritable nom. La Kahina est en effet un surnom que Kateb Yacine, dans cette pièce, a choisi de remplacer par l'authentique identité de la reine berbère : Dihya. Par ce personnage, Kateb Yacine représente le patriotisme et l'opposition farouche aux invasions étrangères. La première figure à avoir résisté aux envahisseurs de la terre algérienne est pour lui une héroïne nationale qu'il réhabilite dans ses écrits. Elle lui permet également d'honorer les femmes qui, elles aussi, ont participé au mouvement de résistance.

Mohammed Khaï-Eddine, écrivain marocain, fait lui aussi, intervenir la Kahina dans ses deux premiers romans (Agadir, et Corps négatif suivi de Histoire d'un bon Dieu), en privilégiant le caractère rebelle du personnage et en le mettant en scène dans des situations contemporaines; la Kahina devient alors le symbole de la résistance à l'ordre établi. Cependant, ces interventions sont brèves et se présentent sous forme de dialogues qui permettent à l'auteur de transmettre à ses contemporains son propre message politique. Par la voix de ce personnage qui est entré dans la légende, Mohammed Khaïr-Eddine dénonce la situation déplorable de ses compatriotes et critique le pouvoir central. L'utilisation de cette figure symbole de la résistance berbère accentue le message. Dans son premier roman, Mohammed Khaïr-Eddine la présente même comme une communiste. Son statut de femme mythique permet à l'auteur de lui faire tenir certains propos qui ne pourraient être exprimés par d'autres personnages. Elle symbolise la résistance et veut assassiner le roi :

Vos royautés cent fois interdites, vos danses d'éclipses, vos interruptions dans le galop du sang, vos crimes, vos fastes sans basilic, sans vraie fête le peuple opprimé de faim molesté d'astres intangibles pérégrinant aux confins du néant, vos soldats mandataires vos bistrots et vos corrupteurs nous ont réveillés par leur vaste chahut. Nous connaissons bien ton rôle. Tu devras donc cesser de lutter pour une cause nuisible. Faire venir le peuple ici. Nous lui inculquerons notre vérité et notre angoisse?.

Dans Corps négatif, il est d'abord question d'une femme anonyme : ce n'est qu'après la prise de parole que le personnage se présente comme la Kahina :

Je m’appelle la brûlée-vive [...], je suis l'aigle femme [...]. Je m’appelle la tuéevive.

[...] de-son-visage-de-sarrasin-et-de-violette Kahina ${ }^{\mathrm{I}}$.

Les deux évocations de ce personnage historique en font un mythe par l'irréalité qui semble l'entourer : ses apparitions et disparitions - éclipses et

9. M. Khaï-Eddine, Agadir, Seuil, I967, p. 59.

ı. M. Khaïr-Eddine, Corps négatif suivi de Histoire d’un bon Dieu, Seuil, 1968, p. IIo-II6. 
surgissements - sont subites et semblent même relever de la magie. La forme même de l'écrit, qui associe le récit à la poésie, renforce la particularité de ce personnage venu de nulle part et allant nulle part. Si elle véhicule une figure positive dans la mesure où elle représente la résistance et la révolte, la Kahina, comme personnage symbolique, renvoie à des réalités différentes, politiques (berbère, maghrébin...) ou féministes. Il semble bien que chacun donne à ce personnage le sens qu'il désire en oubliant parfois la réalité historique.

Aïcha Kandicha, quant à elle, jouit d'un nom mythique capable à lui seul de créer un personnage qui réveille tout l'imaginaire marocain et parfois maghrébin. De nombreux écrivains évoquent ce nom qui crée un monde magique, particulier, parfois angoissant. Annie Devergnas-Dieumegard, dans son étude du monde végétal, animal et magique dans la littérature marocaine ${ }^{\text {II }}$ relève les romans marocains qui font appel à ce personnage mythique dans leur narration. Nous nous pencherons plus particulièrement sur le roman de Tahar Ben Jelloun, Harrouda. Dans ce premier roman, l'auteur marocain a introduit cette figure féminine mythique et particulièrement caractéristique du Maroc grâce au personnage d'Harrouda. Bien qu'il la nomme ainsi, le nom de Kandicha est également inséré dans la narration :

femme à l'âge interchangeable, ex-sirène de la Méditerranée, veuve de l'Ogre de Fass, maitresse de l'araignée Kandisha... cherche compagnon et complice pour libérer un territoire, enlever les femmes du harem de Moulay Idriss et dresser les oiseaux du socco chico... ${ }^{\text {I2 }}$

Harrouda est protéiforme, oscillant entre la figure de la mère et de la prostituée, entre la prêtresse et le démon. Cette femme est à la fois la prostituée qui incarne l'interdit et la femme surnaturelle qui fait naître tous les fantasmes et apparaît comme un danger. Comme Kandicha, elle est associée au monde de la nuit et de la sexualité. Tahar Ben Jelloun fait d'elle l'incarnation des fantasmes enfantins, puisqu' elle symbolise la femme par qui tout est possible :

Mais qui ose?

Qui ose parler de cette femme?

Harrouda n'apparaît que le jour. Le soir elle disparaît quelque part dans une grotte. Loin de la ville. Loin de ses trappes. Elle rétablit son pacte avec l'Ogre et se donne à lui. Toute à lui. Sans lui faire payer le moindre râle. Nous restons persuadés qu'au milieu de la nuit elle lui échappe pour faire les terrasses. Elle surveille notre sommeil et préside nos rêves. La peur de la rencontrer seule manipule nos désirs échangés ${ }^{13}$.

II. A. Devergnas-Dieumegard, Chiens errants et arganiers. Le monde naturel dans l'imaginaire des écrivains marocains de langue française, L'harmattan, coll. "Critiques littéraires", 2003, p. 433.

I2. T. Ben Jelloun, Harrouda, Denoël, I973, p. I7I.

13. Ibid., p. I4. 
Le caractère magique et surnaturel d'Harrouda est, naturellement, emprunté à Kandicha. Ce personnage non conformiste permet d'aborder les questions taboues telles que le corps, le sexe, la femme. Elle permet de créer un monde à part, au-delà de ce qui est connu.

Aïcha Kandicha, dont le mythe est principalement populaire et dont l'histoire a été transmise par les contes et l'oralité, a, beaucoup plus que la Kahina, inspiré d'autres expressions artistiques telles que le cinéma ou la musique. Comme en littérature, au cinéma, Aïcha Kandicha est une figure qui apparaît en filigrane pour souligner le mystère, la magie, le surnaturel. Elle joue un rôle central dans deux films récents : Elle d'Ibrahim Chakiri (2006) et Kandisha de Jérôme Cohen-Olivar (2010). Dans Elle, le personnage principal rencontre une inconnue qui va entrer dans sa vie pour le persécuter. En essayant de déterminer qui est cette femme, tout l'amène à reconnaître en elle la Kandicha. Le deuxième film pose la question de l'existence véritable de cette femme : une femme, accusée du meurtre de son mari qui la violentait, dit avoir été vengée par Aïcha Kandicha. L'avocate qui la défend doit se faire une idée sur la question. Ces deux films permettent de cerner les deux grandes questions que suscite ce personnage mythique : a-t-elle existé? A-t-elle eu pour rôle de persécuter les hommes afin de les rendre fous? Les Gnawas rendent également hommage musicalement à Aïcha Kandicha de plus en plus ouvertement par des évocations. Nous citerons par exemple une chanson interprétée par Hamid el Kasri. Jil Jillala, le célèbre groupe musical marocain des années 1970, lui a également consacré une chanson intitulée «Lella Aïcha».

Toutes ces traces de ces deux personnages mythiques dans différentes expressions artistiques montrent l'attachement des populations à des figures féminines qui font désormais partie de la mémoire collective et qui continuent de la nourrir. Bien qu'elles soient très proches à l'origine, du fait de leurs actes historiques, la Kahina et Aïcha Kandicha ont désormais chacune leur mythe propre qui a fait son ouvre dans la mémoire collective. C'est d'ailleurs là le fait du mythe, comme le souligne Denise Brahimi au sujet de la Kahina, dans une étude qui lui est consacrée dans Femmes arabes et sæurs musulmanes:

La Kahina échappe aux hommes et à l'histoire pour entrer dans le mythe : c'est une autre manière de dire qu'elle ne correspond plus à une vérité vécue et observable parmi les femmes de son pays mais qu'elle survit avec la force d'une idée, d'autant plus indestructible qu'elle est profondément enfouie ${ }^{14}$.

I4. D. Brahimi, Femmes arabes et sours musulmanes, Tierce, I984. 